\title{
Comparative investigations of nonlinear and linear observers for a highly manoeuvrable target in sliding mode guidance
}

\author{
Y. WANG ${ }^{1}$, M. SUN ${ }^{2,3 *}, \mathrm{~S} . \mathrm{DU}^{4}$, and Z. $\mathrm{CHEN}^{2,3}$ \\ ${ }^{1}$ AVIC LEIHUA Electronic Technology Research Institute, Wuxi 214063, China \\ ${ }^{2}$ College of Computer and Control Engineering, Nankai University,Tianjin 300350, China \\ ${ }^{3}$ Tianjin Key Laboratory of Intelligent Robotics, Nankai University, Tianjin 300350, China \\ ${ }^{4}$ Department of Mechanical Engineering, Tshwane University of Technology, Pretoria 0001, South Africa
}

\begin{abstract}
Target manoeuvre is one of the key factors affecting guidance accuracy. To intercept highly maneuverable targets, a second-order sliding-mode guidance law, which is based on the super-twisting algorithm, is designed without depending on any information about the target motion. In the designed guidance system, the target estimator plays an essential role. Besides the existing higher-order sliding-mode observer (HOSMO), a first-order linear observer (FOLO) is also proposed to estimate the target manoeuvre, and this is the major contribution of this paper. The closed-loop guidance system can be guaranteed to be uniformly ultimately bounded (UUB) in the presence of the FOLO. The comparative simulations are carried out to investigate the overall performance resulting from these two categories of observers. The results show that the guidance law with the proposed linear observer can achieve better comprehensive criteria for the amplitude of normalised acceleration and elevator deflection requirements. The reasons for the different levels of performance of these two observer-based methods are thoroughly investigated.
\end{abstract}

Key words: guidance law, second-order sliding mode, super-twisting algorithm, linear observer, target manoeuvre.

\section{Introduction}

Classical proportional navigation (PN) is widely used in practice, but it is the optimal guidance law only for intercepting one non-manoeuvrable or weakly manoeuvrable target [1]. To intercept highly manoeuvrable targets, the miss distance would increase considerably if the traditional PN law was employed and the performance would be unacceptable. Therefore, several improved PN laws have been proposed [2, 3]. These guidance algorithms can reduce the miss distance, but the target manoeuvre information must be known in advance. The optimal guidance investigation is also a hot debated topic $[4,5]$. Nonetheless, the target velocity variation was not considered in [4], and the optimal guidance law from [5] can be realised only in the presence of accurate knowledge of the target manoeuvre. An $H_{\infty}$ guidance law was proposed in [6], wherein the target manoeuvre was treated as an unpredictable disturbance, and a favourable interception performance for arbitrary scenarios of target manoeuvres could be guaranteed if the target acceleration is bounded; however, the huge computational complexity is a severe constraint to the implementation of this $H_{\infty}$ method. These deficiencies limit the practical applications of the above strategies.

Since the sliding mode control (SMC) method was firstly proposed, it has attracted a lot of researchers worldwide [7, 8] due to its robustness to parameter perturbations and external

\footnotetext{
*e-mail: smw_sunmingwei@163.com
}

matched disturbances. However, the chattering phenomenon is the major obstacle for the implementation of SMC in practice, and a number of methods have been proposed to reduce the chattering. Levant first proposed the higher-order sliding mode (HOSM) control method to attenuate the chattering [9-12]. The second-order sliding mode control is the most widely used HOSM method in which the super-twisting algorithm is utilised to attenuate the differentiable disturbance and ensure the finite-time stability $[13,14]$. To intercept highly manoeuvrable targets, the second-order sliding mode methods were utilised in [13] and [14], based on the nonlinear observers, which are used to check the uncertainty caused by target manoeuvres. However, in many simulations, it is readily observed that the guidance commands (acceleration) generated by the proposed guidance laws of [13] and [14] exhibit the properties of oscillation with a large amplitude. Comparing the fundamental guidance component with the observer component, the latter contributes to this phenomenon much more. Hence, an effective estimation approach is urgently needed to deal with highly manoeuvrable targets.

Han proposed a novel philosophy, namely the active disturbance rejection control (ADRC) algorithm [15, 16], which has attracted wide attention in the past decade. The core of ADRC is an extended state observer (ESO) that can accurately observe the total disturbance. Han originally presented the ESO in a nonlinear form [15], which is too complicated to tune, and is difficult to be implemented. In [16], a linear ESO (LESO) was proposed in terms of frequency bandwidth, which provides a useful design approach in practical applications. Although this 
linear observer is simple and there is only one tunable parameter, it has demonstrated its effectiveness when applied to control diverse nonlinear systems [17-21]. In addition, a binary distillation column can be controlled by using a linear proportional-integral observer in [22]. In [23], a polynomial controller was proposed for a quad-rotor unmanned aerial vehicle (UAV) combined with a linear observer to deal with bounded uncertainties and disturbances. Therefore, a linear observer is still a useful tool in coping with nonlinear control problems.

In this research, a second-order sliding mode guidance law is designed based on the super-twisting algorithm to intercept highly manoeuvrable targets. To estimate the target manoeuvre and compensate it, a first-order linear observer (FOLO) is proposed in the guidance law, which is the major contribution of this study. The advantage of our work is that it has significantly weak requirements of elevator deflection in contrast with the nonlinear observer-based method in [13]. The comprehensive stability of the proposed sliding mode guidance law is also investigated when it is combined with the FOLO. In the numerical simulations, the effects of the proposed method and the original nonlinear observer-based method [13] are comparatively illustrated. The reason behind the different performance for these two observer-based methods is thoroughly investigated.

The remainder of this paper is organised as follows. In section 2, a mathematical model of the guidance problem is presented. The second-order sliding mode guidance law based on the super-twisting algorithm and the FOLO is proposed in section 3 . The stability analysis is presented in section 4 . Section 5 furnishes the comparative simulation results. Finally, the concluding remarks are given in the last section.

\section{Formulation of missile-target engagement kinematics}

The geometry of planar interception is depicted in Fig. 1. The engagement kinematics are (as presented in $[13,14]$ )

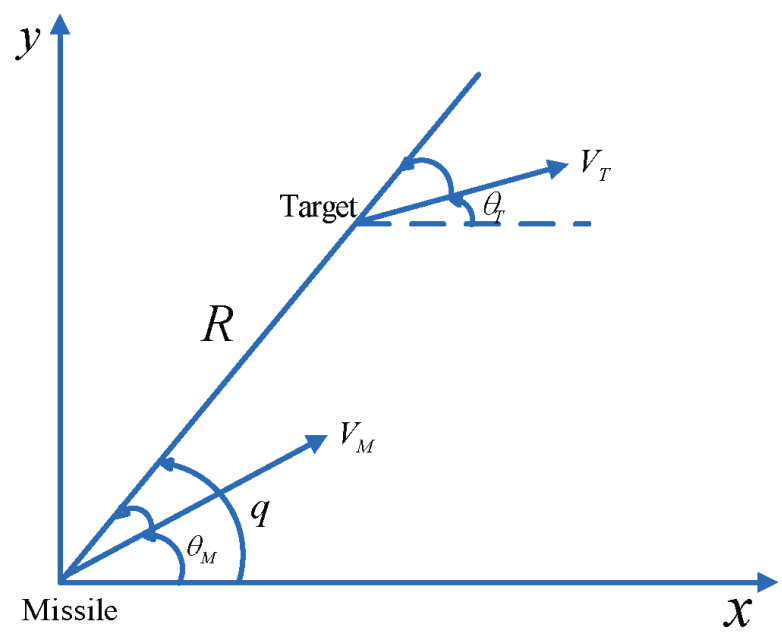

Fig. 1. Engagement geometry of target and missile

$$
\begin{aligned}
& \dot{R}=V_{\Pi}, \\
& \dot{V}_{\Pi}=V_{\perp}^{2} / R+a_{T_{\Pi}}+a_{M} \sin \left(q-\theta_{M}\right), \\
& \dot{q}=V_{\perp} / R, \\
& \dot{V}_{\perp}=-V_{\Pi} V_{\perp} / R+a_{T_{\perp}}-a_{M} \cos \left(q-\theta_{M}\right),
\end{aligned}
$$

where $q$ is the line-of-sight (LOS) angle; $R$ is the range along LOS; $\theta_{M}$ and $\theta_{T}$ are the flight path angles of the missile and the target, respectively; $V_{\Pi}$ and $V_{\perp}$ are the relative velocity components parallel with the LOS and normal to the LOS, respectively; $a_{T_{\Pi}}$ and $a_{T_{\perp}}$ are the target acceleration along and normal to the LOS, respectively, and $a_{T_{\Pi}}=a_{T} \sin \left(q-\theta_{T}\right)$ and $a_{T_{\perp}}=a_{T} \cos \left(q-\theta_{T}\right) ; a_{M}$ and $a_{T}$ are the normalized accelerations of the missile and the target, respectively.

\section{Guidance law design}

3.1. Preliminary of super-twisting algorithm. Consider the following sliding mode:

$$
\dot{\sigma}=f(t)+u
$$

where $\sigma$ is the sliding variable and $u$ is the control variable, $f$ is a sufficiently smooth uncertain function [13]. The disturbance term $f$ is to be cancelled by means of a special observer to be developed.

Lemma 1. After [12, 24], consider the system (2) without the uncertain term or $f=0$ and select the control as:

$$
u=-\alpha_{1}|\sigma|^{1 / 2} \operatorname{sign}(\sigma)-\alpha_{2} \int \operatorname{sign}(\sigma) d \tau,
$$

where $\alpha_{1}$ and $\alpha_{2}$ are real positive constants. Then $\dot{\sigma}=\sigma=0$ can be achieved in a finite time.

Combining (2) and (3) yields

$$
\left\{\begin{array}{l}
\dot{\sigma}=-\alpha_{1}|\sigma|^{1 / 2} \operatorname{sign}(\sigma)+\bar{\sigma} \\
\dot{\bar{\sigma}}=-\alpha_{2} \operatorname{sign}(\sigma)
\end{array},\right.
$$

which is the super-twisting algorithm.

Refer to $[12,13]$ for the proof of Lemma 1 . Note that (3) is a special nonlinear PI controller with $\alpha_{1}$ and $\alpha_{2}$ as its proportional and integral coefficients, respectively.

3.2. Second-order sliding mode guidance law formulation. The guidance objective is to achieve a hit-to-kill that means $R\left(t_{f}\right)=0$ where $t_{f}$ is the impact time. In $[13,25]$, this objective desires $R<0$ or $V_{\Pi}<0$ in the guidance. Substituting (1c) into (1b) yields

$$
\dot{V}_{\Pi}=R \dot{q}^{2}+a_{T_{\Pi}}+a_{M} \sin \left(q-\theta_{M}\right) .
$$

The term $R \dot{q}^{2}$ in (5) represents the centrifugal acceleration, which rapidly grows as the LOS keeps rotating. It provides 
almost instant reverse in $V_{\Pi}$ direction when $R$ becomes small. Therefore, zeroing $\dot{q}$ is a useful strategy to avoid the reverse of $V_{\Pi}$. On the other hand, zeroing $\dot{q}$ implies that $V_{\perp} \rightarrow 0$ according to (1c). In fact, zeroing $\dot{q}$ was the primary goal for many developed guidance laws. The control task in this case is to counteract $a_{T_{\perp}}$, which attempts to drive $\dot{q}$ to be divergent.

Here, a relaxed requirement is considered. If $\dot{q}$ is allowed to grow and not faster than $\frac{1}{R^{\beta}}(0<\beta<1$ as $R \rightarrow 0)$, one can obtain $V_{\perp}=R \dot{q} \leq R \frac{1}{R^{\beta}}=R^{1-\beta}$ as $R \rightarrow 0$. It should be noted that $R \dot{q}^{2}$ should be bounded by $F$ as $R \rightarrow 0$ in (5), otherwise the reverse in $V_{\Pi}$ is inevitable. Therefore, we must make sure that $R \dot{q}^{2} \leq F$ as $R \rightarrow 0$ and

$$
\dot{q} \leq \frac{\sqrt{F}}{R^{1 / 2}}=\frac{1}{R^{\beta}}, \quad \beta \leq \frac{1}{2} .
$$

Based on (6), a suitable LOS rate is chosen as

$$
\dot{q}=\frac{c_{0}}{R^{1 / 2}}
$$

where $0<c_{0}<1$. Combining (1c) and (7) yields

$$
V_{\perp}=c_{0} \sqrt{R} \text {. }
$$

The consequent task is to stabilize the system $(1 \mathrm{a}-1 \mathrm{~d})$ on the manifold $V_{\perp}-c_{0} \sqrt{R}=0$. Define the sliding surface as

$$
s=V_{\perp}-c_{0} \sqrt{R} .
$$

Differentiating (9) gives rise to

$$
\dot{s}=\dot{V}_{\perp}-\frac{c_{0} \dot{R}}{2 \sqrt{R}} .
$$

Substituting (1d) into (10) yields

$$
\dot{s}=-\dot{R} \dot{q}-\cos \left(q-\theta_{M}\right) a_{M}+a_{T_{\perp}}-\frac{c_{0} \dot{R}}{2 \sqrt{R}} .
$$

In (11), $a_{T_{\perp}}$ can be regarded as an external disturbance and we can define

$$
f(t)=a_{T_{\perp}}
$$

The reaching law of $s$ is designed by the super-twisting algorithm as

$$
\dot{s}=-\alpha_{1}|s|^{1 / 2} \operatorname{sign}(s)-\int_{0}^{t} \alpha_{2} \operatorname{sign}(s) d t .
$$

Piecing (11), (12), and (13) together yields

$$
\begin{aligned}
\bar{a}_{M}= & \frac{1}{\cos \left(q-\theta_{M}\right)} \cdot \\
& {\left[\begin{array}{l}
-\left(-\alpha_{1}|s|^{1 / 2} \operatorname{sign}(s)-\right. \\
\left.\int_{0}^{t} \alpha_{2} \operatorname{sign}(s) d t\right)-\dot{R} \dot{q}-\frac{c_{0} \dot{R}}{2 \sqrt{R}}+f(t)
\end{array}\right] }
\end{aligned}
$$

This is the desired guidance command that is consistent with the proposed algorithm in [13], wherein the unknown term $f(t)$ was estimated and compensated by a HOSM observer (HOSMO) [13] which is formulated as

$$
\left\{\begin{array}{l}
\dot{\bar{z}}_{0}=v_{0}-a_{M} \cos \left(q-\theta_{M}\right)-\dot{R} \dot{q}-c_{0} \dot{R} /(2 \sqrt{R}) \\
v_{0}=-2 L^{1 / 3}\left|\bar{z}_{0}-s\right|^{2 / 3} \operatorname{sign}\left(\bar{z}_{0}-s\right)+\bar{z}_{1} \\
\dot{\bar{z}}_{1}=v_{1} \\
v_{1}=-1.5 L^{1 / 2}\left|\bar{z}_{1}-v_{0}\right|^{1 / 2} \operatorname{sign}\left(\bar{z}_{1}-v_{0}\right)+\bar{z}_{2} \\
\bar{z}_{2}=-1.1 L \operatorname{sign}\left(\bar{z}_{2}-v_{1}\right) \\
f(t)=\bar{z}_{1}
\end{array}\right.
$$

where $\bar{z}_{0}, \bar{z}_{1}$ and $\bar{z}_{2}$ are the estimations of $s, f(t)$ and $\dot{f}(t)$, respectively. Moreover, $L$ is the observer gain and it is the known Lipshitz constant [13] of $\dot{f}(t)$. In other words, $|\ddot{f}(t)| \leq L$. According to (15), the error dynamics of HOSMO can be obtained as

$$
\left\{\begin{aligned}
\dot{\Delta}_{0} & =-2 L^{1 / 3}\left|\Delta_{0}\right|^{2 / 3} \operatorname{sign}\left(\Delta_{0}\right)+\Delta_{1} \\
\dot{\Delta}_{1} & =-1.5 L^{1 / 2}\left|\Delta_{1}-\dot{\Delta}_{0}\right|^{1 / 2} \operatorname{sign}\left(\Delta_{1}-\dot{\Delta}_{0}\right)+\Delta_{2} \\
\dot{\Delta}_{2} & =-1.1 L \operatorname{sign}\left(\Delta_{2}-\dot{\Delta}_{1}\right)-\ddot{f} \\
& \in-1.1 L \operatorname{sign}\left(\Delta_{2}-\dot{\Delta}_{1}\right)+[-L, L]
\end{aligned}\right.
$$

where $\Delta_{0}=\bar{z}_{0}-s, \Delta_{1}=\bar{z}_{1}-f$, and $\Delta_{2}=\bar{z}_{2}-\dot{f}$. Define $\xi(\Delta): R^{3} \rightarrow R^{3} \quad$ and $\xi(\Delta)=\left[\xi_{1}(\Delta), \xi_{2}(\Delta), \xi_{3}(\Delta)\right]^{\mathrm{T}}=\left[\dot{\Delta}_{1}, \dot{\Delta}_{2}, \dot{\Delta}_{3}\right]^{\mathrm{T}} \quad$ with $\Delta=\left[\Delta_{0}, \Delta_{1}, \Delta_{2}\right]^{\mathrm{T}}$. Note that the function $\xi(\Delta)$ represents the right-hand side of (44), and we have the following result.

Theorem 1. The function $\xi(\Delta)$ is homogeneous of degree $\bar{q}=-1$ with the dilation $d_{\kappa}:\left(\Delta_{0}, \Delta_{1}, \Delta_{2}\right) \rightarrow\left(\kappa^{m_{0}} \Delta_{0}, \kappa^{m_{1}} \Delta_{1}, \kappa^{m_{2}} \Delta_{2}\right)$ and the homogeneity weights $m_{i}$ for $\Delta_{i}, i=0,1,2$ as

$$
m_{0}=3, \quad m_{1}=2, \quad m_{2}=1,
$$

and $\xi(\Delta)=\kappa^{-\bar{q}} d_{\kappa}^{-1} \xi\left(d_{\kappa} \Delta\right)$ for any $\kappa>0$.

Proof. For the dynamics of $\Delta_{0}$, one has

$$
\begin{aligned}
& \kappa^{-\bar{q}} d_{\kappa}^{-1} \xi_{0}\left(d_{\kappa} \Delta\right) \\
& =\kappa^{-\left(-1+m_{0}\right)}\left[-2 L^{1 / 3}\left|\kappa^{m_{0}} \Delta_{0}\right|^{2 / 3} \operatorname{sign}\left(\Delta_{0}\right)+\kappa^{m_{1}} \Delta_{1}\right] \\
& =\kappa^{-2}\left[-2 L^{1 / 3}\left|\kappa^{3} \Delta_{0}\right|^{2 / 3} \operatorname{sign}\left(\Delta_{0}\right)+\kappa^{2} \Delta_{1}\right] \\
& =-2 L^{1 / 3}\left|\Delta_{0}\right|^{2 / 3} \operatorname{sign}\left(\Delta_{0}\right)+\Delta_{1} \\
& =\xi_{0}(\Delta) .
\end{aligned}
$$

Based on the first equation of (16), one can obtain

$$
\Delta_{1}-\dot{\Delta}_{0}=2 L^{1 / 3}\left|\Delta_{0}\right|^{2 / 3} \operatorname{sign}\left(\Delta_{0}\right)
$$


and

$$
\begin{aligned}
& =-\left.\left.1.5 L^{1 / 2}\left|2 L^{1 / 3}\right| \Delta_{0}\right|^{2 / 3} \operatorname{sign}\left(\Delta_{0}\right)\right|^{1 / 2} \operatorname{sign}\left(\Delta_{0}\right)+\Delta_{2} \\
& =-1.5 \sqrt{2} L^{2 / 3}\left|\Delta_{0}\right|^{1 / 3} \operatorname{sign}\left(\Delta_{0}\right)+\Delta_{2} .
\end{aligned}
$$

Then, we have

$$
\begin{aligned}
& \kappa^{-\bar{q}} d_{\kappa}^{-1} \xi_{1}\left(d_{\kappa} \Delta\right) \\
& =\kappa^{-\left(-1+m_{1}\right)}\left[-1.5 \sqrt{2} L^{2 / 3}\left|\kappa^{m_{0}} \Delta_{0}\right|^{1 / 3} \operatorname{sign}\left(\Delta_{0}\right)+\kappa^{m_{2}} \Delta_{2}\right] \\
& =\kappa^{-1}\left[-1.5 \sqrt{2} L^{2 / 3}\left|\kappa^{3} \Delta_{0}\right|^{1 / 3} \operatorname{sign}\left(\Delta_{0}\right)+\kappa \Delta_{2}\right] \\
& =-1.5 \sqrt{2} L^{2 / 3}\left|\Delta_{0}\right|^{1 / 3} \operatorname{sign}\left(\Delta_{0}\right)+\Delta_{2} \\
& =\xi_{1}(\Delta) .
\end{aligned}
$$

Therefore, the homogeneity equations $\xi(\Delta)=\kappa^{-\bar{q}} d_{\kappa}^{-1} \xi\left(d_{\kappa} \Delta\right)$, $i=0,1,2$ are established, and the HOSMO is homogeneous. This completes the proof.

Note that (15) is essentially a nonlinear observer with fractional power. It should be pointed out that a uniform convergence cannot be achieved for a homogeneous system [26]. In this paper, a linear observer is adopted to estimate $f(t)$.

\subsection{Target motion estimation by using FOLO}

Denote the following known information in (11) as

$$
f_{0}(t)=-\dot{R} \dot{q}-\frac{c_{0} \dot{R}}{2 \sqrt{R}},
$$

and then, (11) can be reformulated as

$$
\dot{s}=f_{0}(t)+f(t)-\cos \left(q-\theta_{M}\right) a_{M},
$$

which can be described in a state-space form as

$$
\left\{\begin{array}{l}
\dot{x}_{1}=x_{2}+b \bar{u} \\
\dot{x}_{2}=w \\
y=x_{1}
\end{array} .\right.
$$

Here,

$$
\left\{\begin{array}{l}
x_{1}=s \\
x_{2}=f(t) \\
b=-\cos \left(q-\theta_{M}\right) . \\
\bar{u}=\frac{f_{0}(t)}{b}+a_{M}
\end{array}\right.
$$

$x_{2}$ is denoted as an extended state and $w$ is its derivative; $w$ can be regarded as a jerk [27] that generates $x_{2}$ and terminates the description of the state-space. Note that $\bar{u}$ is a virtual guidance command, which is used to simplify the design process, and it contains all of the known information of the derivative of $s$.
It is now necessary to estimate $x_{2}$. Since $x_{1}$ is available, a reduced-order observer can be employed to decrease the phase lag. For the $x_{2}$ subsystem

$$
\dot{x}_{2}=w
$$

we have

$$
x_{2}=\dot{x}_{1}-b \bar{u}
$$

according to (24). In terms of (26) and (27), the observer for $x_{2}$ can be written as

$$
\dot{z}_{2}=l\left(\dot{x}_{1}-b \bar{u}-z_{2}\right)
$$

where $z_{2}$ is the estimation for $x_{2}$, and $l$ is the observer gain. However, since $\dot{x}_{1}$ is not available, we define a new variable

$$
z_{c}=z_{2}-l x_{1}
$$

in order to reformulate (28) as

$$
\dot{z}_{c}=-l z_{2}-l b \bar{u} \text {. }
$$

Note that (29-30) can only estimate $x_{2}$ with its order as 1 , therefore it is a first-order linear observer (FOLO) from the second-order extended system (24). The observer gain $l$ can be denoted as the observer bandwidth $\omega_{0}$. With a well-tuned observer, one can obtain $z_{2} \approx x_{2}$. Hence $z_{2}$ can be utilised to mitigate the target manoeuvre in a straightforward way: the desired guidance command (14) can be implemented as

$$
\begin{aligned}
a_{M}= & \frac{1}{\cos \left(q-\theta_{M}\right)}\left[-\left(-\alpha_{1}|s|^{1 / 2} \operatorname{sign}(s)\right.\right. \\
& \left.\left.-\int_{0}^{t} \alpha_{2} \operatorname{sign}(s) d t\right)-\dot{R} \dot{q}-c_{0} \dot{R} /(2 \sqrt{R})+z_{2}\right] .
\end{aligned}
$$

In order to avoid $\left|q-\theta_{M}\right|=\pi / 2$, a defending measure is setting $\left|q-\theta_{M}\right|=\mu$ when $\left|q-\theta_{M}\right| \geq \mu$. Here, $\mu$ is constant and just less than $\pi / 2$, for example $\mu=\pi / 2 \times 80 / 90$.

Remark 1. Since $V_{T}$ can be calculated via $V_{\perp}=R \dot{q}$ with measurable $R$ and $\dot{q}, x_{1}$ can be obtained in real time as $x_{1}=V_{\perp}-c_{0} \sqrt{R}$, where $c_{0}$ is a given positive constant. Therefore $x_{1}$ is available. Furthermore, the observation of $x_{1}$ is not needed anymore, and only the extended state $x_{2}$ needs to be estimated by (29-30). Although this observer contains two equations (one is differential and the other is algebraic), its order is 1 because the order of an observer is defined as its differential order. In this case, only $x_{2}$ is estimated for the $\left(x_{1}, x_{2}\right)$ system wherein a second-order observer is generally needed. From this point of view, we can declare it is a reduced-order observer. In such a way, the phase lag triggered by the estimator can be reduced and the proposed method can enhance the closed-loop robustness effectively.

Remark 2. In (31), the flight path angle $\theta_{M}$ can be calculated by using the horizontal velocity $V_{x}$ and vertical velocity $V_{y}$ from 
the on-board inertial navigation system (INS). Nevertheless, it is well known that the sole INS vertical position solution is dynamically exponentially unstable since the computer must use the vertical position to compute and compensate the measured accelerations for normal gravity. This problem can be solved by using some improved compensation algorithms. The gravity can be fixed as a constant, because the terminal guidance period is generally quite short. The anomalous gravity field map can also be used in a map-matching mode to bound the vertical velocity error [28]. Through these ways, a sufficiently accurate vertical velocity can be obtained, and the flight path angle $\theta_{M}$ can be obtained as

$$
\theta_{M}=\arctan \left(V_{y} / V_{x}\right)
$$

Remark 3. Although there are two sign functions in (31), the guidance command is still continuous (not smooth) [13]. The first sign function ensures that the square root function makes sense for each real number $s$ while still maintaining the sign thereof. The second sign function takes effect with an integral operator; hence its role is demonstrated in a continuously incremental form. It is remarkable that these fundamental characteristics of the second-order sliding mode approach can check the chattering phenomenon by eliminating the direct sign terms.

\section{Stability analysis}

In this section, the stability of the proposed guidance law is analysed.

Substituting the guidance law (31) into (11), the closed-loop system is given as

$$
\dot{s}=-\alpha_{1}|s|^{1 / 2} \operatorname{sign}(s)-\int_{0}^{t} \alpha_{2} \operatorname{sign}(s) d t+f(t)-z_{2},
$$

which can be rewritten as

$$
\left\{\begin{array}{l}
\dot{v}_{1}=-\alpha_{1}\left|v_{1}\right|^{1 / 2} \operatorname{sign}\left(v_{1}\right)+v_{2}+e \\
\dot{v}_{2}=-\alpha_{2} \operatorname{sign}\left(v_{1}\right)
\end{array}\right.
$$

where $v_{1}=s, v_{2}=-\int_{0}^{t} \alpha_{2} \operatorname{sign}(s) d t$, and $e=f(t)-z_{2}$ represents the observer error.

Choose the Lyapunov function of [13]

$$
V_{1}=\frac{v_{2}^{2}}{2}+\int_{0}^{v_{1}} \alpha_{2} \operatorname{sign}(t) d t
$$

The derivative of $V_{1}$ is

$$
\begin{aligned}
\dot{V}_{1}= & v_{2} \dot{v}_{2}+\alpha_{2} \operatorname{sign}\left(v_{1}\right) \dot{v}_{1} \\
= & -v_{2} \alpha_{2} \operatorname{sign}\left(v_{1}\right)+\alpha_{2} \operatorname{sign}\left(v_{1}\right) \\
& \left(e-\alpha_{1}\left|v_{1}\right|^{1 / 2} \operatorname{sign}\left(v_{1}\right)+v_{2}\right) \\
\leq & -\alpha_{1} \alpha_{2}\left|v_{1}\right|^{1 / 2}+\alpha_{2}|e| .
\end{aligned}
$$

With respect to (36), we cannot immediately confirm whether the system is stable when employing the Lyapunov function in [13].

Because the target manoeuvrability is finite, it can be effectively captured by a sufficiently fast observer. Note that strong manoeuvrability generally means a large amplitude instead of a high-frequency variation in order for the target acceleration to evade interception in reality. In other words, $f(t)$ can be regarded as a low-frequency signal and it is reasonable to give the following assumption.

Assumption 1. The derivative of the target normal acceleration $a_{T_{\perp}}$ is assumed to be bounded as

$$
|\dot{f}(t)| \leq \varepsilon_{f}
$$

where $\varepsilon_{f}$ is an unknown constant.

Theorem 2. The sliding mode $v_{1}$ and the observer error $e$ in the closed-loop system (33) or (34) are uniformly ultimately bounded (UUB) [29] as

$$
\left|v_{1}\right| \leq \varepsilon^{2}\left(\alpha_{1} \alpha_{2}\right)^{-2}
$$

and

$$
|e| \leq \sqrt{\varepsilon / \omega_{o}}+\left(\alpha_{2}+\varepsilon_{f}\right) /\left(2 \omega_{o}\right)
$$

respectively, when the interception time is sufficiently large, where

$$
\varepsilon=\left(\alpha_{2}+\varepsilon_{f}\right)^{2}\left(4 \omega_{o}\right)^{-1}
$$

Proof. For the closed-loop system (33) or (34), choose an improved Lyapunov function

$$
V_{2}=\frac{v_{2}^{2}}{2}+\int_{0}^{v_{1}} \alpha_{2} \operatorname{sign}(t) d t+\frac{e^{2}}{2} .
$$

Note that $\int_{0}^{v_{1}} \alpha_{2} \operatorname{sign}(t) d t \geq 0$, so $V_{2}$ is positive definite. According to (36), the derivative of $V_{2}$ can be derived as

$$
\dot{V}_{2} \leq-\alpha_{1} \alpha_{2}\left|v_{1}\right|^{1 / 2}+\alpha_{2}|e|+e \dot{e}
$$

Combining (30) and the differential formula of (29) yields

$$
\left\{\begin{array}{l}
\dot{z}_{c}=\dot{z}_{2}-\omega_{o} \dot{x}_{1} \\
\dot{z}_{c}=-\omega_{o} z_{2}-\omega_{o} b \bar{u}
\end{array} .\right.
$$

As $\dot{x}_{1}=x_{2}+b \bar{u},(43)$ can be simplified as

$$
\begin{aligned}
\dot{z}_{2} & =\omega_{o}\left(-b \bar{u}-z_{2}+x_{2}+b \bar{u}\right) \\
& =\omega_{o} e .
\end{aligned}
$$


Substituting (37) and (44) into (42) yields

$$
\begin{aligned}
\dot{V}_{2} & \leq-\alpha_{1} \alpha_{2}\left|v_{1}\right|^{1 / 2}+\alpha_{2}|e|+e\left(\dot{f}(t)-\omega_{o} e\right) \\
& \leq-\alpha_{1} \alpha_{2}\left|v_{1}\right|^{1 / 2}+\alpha_{2}|e|+|e| \varepsilon_{f}-\omega_{o} e^{2} \\
& =-\alpha_{1} \alpha_{2}\left|v_{1}\right|^{1 / 2}-\omega_{o} e^{2}+|e|\left(\varepsilon_{f}+\alpha_{2}\right) \\
& \leq-\alpha_{1} \alpha_{2}\left|v_{1}\right|^{1 / 2}-\left(\sqrt{\omega_{o}}|e|-\frac{\alpha_{2}+\varepsilon_{f}}{2 \sqrt{\omega_{o}}}\right)^{2}+\varepsilon
\end{aligned} .
$$

According to the Lyapunov stability theory, the closed-loop system will be stable when $\dot{V}_{2}$ is strictly negative definite. Via some derivations of the terms of the last line in (45), one can obtain $\dot{V}_{2}<0$ with $|e|>\sqrt{\varepsilon / \omega_{o}}+\left(\alpha_{2}+\varepsilon_{f}\right) /\left(2 \omega_{o}\right)$ or $\left|v_{1}\right|>\varepsilon^{2}\left(\alpha_{1} \alpha_{2}\right)^{-2}$. For the sake of simplicity, we merely consider the case of $e$. With the definition of

$$
\bar{c}=\sqrt{\varepsilon / \omega_{o}}+\left(\alpha_{2}+\varepsilon_{f}\right) /\left(2 \omega_{o}\right)
$$

$\dot{V}_{2}$ will be negative in the set $\{|e|>\bar{c}\}$. In this set, $V_{2}$ will decrease monotonically; and $|e|$ will decrease simultaneously until $|e|$ enters $\{|e| \leq \bar{c}\}$, and then $|e|$ will not leave the set $\{|e| \leq \bar{c}\}$ because $\dot{V}_{2}$ is negative on the boundary $|e|=\bar{c}$. Thereafter, it can be concluded that $|e|$ is uniformly ultimately bounded with an ultimate bound $|e| \leq \sqrt{\varepsilon / \omega_{o}}+\left(\alpha_{2}+\varepsilon_{f}\right) /\left(2 \omega_{o}\right)$. Note that (46) represents the ultimate bound of the observer error. The similar analysis can also be applied to $\left|v_{1}\right|$. This completes the proof. In addition, these two compact bounds can be small enough with a sufficiently large $\omega_{0}$.

\section{Numerical simulations}

In this section, several comparative simulation results are presented, including the tail chase and head-on interception scenarios. For comparisons, we perform simulations using the proposed guidance laws in this paper and in [13], where a HOSMO-based sliding mode guidance law was designed. Both methods are almost identical except the observer design.

Assume that the target has a constant velocity of $600 \mathrm{~m} / \mathrm{s}$. The maximal normalised acceleration of the missile is $25 \mathrm{~g}$ and the target manoeuvres are $a_{T}=8 \sin (0.85 t) \mathrm{g}$ and $a_{T}=8 \operatorname{sign}(\sin (0.85 t)) \mathrm{g}$, respectively, where $\mathrm{g}$ is the gravitational constant.

5.1. Aerodynamic interceptor model. The numerical simulations are performed in order to validate the proposed guidance law in conjunction with an aerodynamic missile controlled by a classical three-loop autopilot. The mathematical model of the missile used here describes the dynamics of a highly maneuverable antiaircraft missile around its pitch axis. The longitudinal equations, which govern the missile dynamics, are [19]

$$
\left\{\begin{array}{l}
\dot{Q}=K_{q} M^{2}(t) C_{m}[\alpha(t), \delta(t), M(t)] \\
\dot{\alpha}=K_{\alpha} M(t) C_{n}[\alpha(t), \delta(t), M(t)] \cos (\alpha(t))+Q \\
\dot{M}(t)=\frac{1}{V_{s}}\left[-\left|a_{z}(t)\right| \sin |\alpha(t)|+a_{x} M^{2}(t) \cos (\alpha(t))\right] \\
C_{n}(\alpha, \delta, M)=a_{n} \alpha^{3} k^{3}+b_{n} \alpha|\alpha| k^{2}+ \\
c_{n}(2-M / 3) \alpha k+d_{n} k \delta \\
C_{m}(\alpha, \delta, M)=a_{m} \alpha^{3} k^{3}+b_{m} \alpha|\alpha| k^{2}+ \\
\quad c_{m}(-7+8 M / 3) \alpha k+d_{m} k \delta \\
a_{x}=0.7 P_{0} S C_{a} / m \\
a_{z}=K_{z} M^{2}(t) C_{n}[\alpha(t), \delta(t), M(t)]
\end{array},\right.
$$

where $Q$ is the pitch rate, $\alpha$ is the angle of attack, $M$ is the Mach number, $\delta$ is the elevator deflection angle, $V_{s}$ is the ground ve-

\begin{tabular}{|c|c|c|c|}
\hline Coefficient & Symbol & Value & Unit \\
\hline \multirow{3}{*}{ constant } & $K_{a}$ & 0.02069 & $\mathrm{~s}^{-1}$ \\
\hline & $K_{q}$ & 1.23219 & $\mathrm{~s}^{-2}$ \\
\hline & $K_{z}$ & 0.66623 & $\mathrm{~s}^{-2}$ \\
\hline \multirow{4}{*}{$\begin{array}{c}\text { Force } \\
\text { coefficient }\end{array}$} & $a_{n}$ & 0.000103 & $\operatorname{deg}^{-3}$ \\
\hline & $b_{n}$ & -0.00945 & $\operatorname{deg}^{-2}$ \\
\hline & $c_{n}$ & -0.16960 & $\operatorname{deg}^{-1}$ \\
\hline & $d_{n}$ & -0.0340 & $\operatorname{deg}^{-1}$ \\
\hline \multirow{4}{*}{$\begin{array}{l}\text { moment } \\
\text { coefficient }\end{array}$} & $a_{m}$ & 0.000215 & $\operatorname{deg}^{-3}$ \\
\hline & $b_{m}$ & -0.0195 & $\operatorname{deg}^{-2}$ \\
\hline & $c_{m}$ & 0.051 & $\operatorname{deg}^{-1}$ \\
\hline & $d_{m}$ & -0.206 & $\operatorname{deg}^{-1}$ \\
\hline static pressure at $6096 \mathrm{~m}$ & $P_{0}$ & 46601.6 & $\mathrm{~N} / \mathrm{m}^{2}$ \\
\hline reference area & $S$ & 0.040877 & $\mathrm{~m}^{2}$ \\
\hline Mass & $m$ & 204.01 & $\mathrm{~kg}$ \\
\hline speed of sound & $v_{s}$ & 315.89 & $\mathrm{~m} / \mathrm{s}$ \\
\hline drag coefficient & $C_{a}$ & -0.3 & 1 \\
\hline
\end{tabular}
locity, $C_{n}$ and $C_{m}$ are the aerodynamic force and moment coefficients, respectively, $a_{x}$ and $a_{z}$ are the longitudinal acceleration and vertical acceleration, respectively. The elevator model is

$$
\dot{\delta}=-\omega_{a} \delta(t)+\omega_{a} \delta_{c}(t)
$$

where $\delta_{c}$ is the control input. Here, the elevator amplitude saturation is $-25^{\circ} \leq \delta \leq 25^{\circ}$ The control task is to ensure the missile vertical acceleration $a_{z}$ to track the real guidance command $a_{M}$, using the rudder deflection angle $\delta$. The parameters of the aerodynamic model are summarised in Table 1 . The operating

Table 1

Characteristic parameters 
range is $-20^{\circ} \leq \alpha \leq 20^{\circ}$ and $1.5 \leq M \leq 3$. The three-loop acceleration autopilot $[30]$ is

$$
\delta_{c}=\int\left[k_{i}\left(k_{p} a_{M}-a_{z}\right)+k_{w} Q\right] d t+k_{d} Q .
$$

5.2. Tuning process. Firstly, the parameters of the guidance law need to be tuned. Similar to the proportional gain in the PI controller, faster convergence of the sliding mode in (13) can be achieved with a larger $\alpha_{1}$, as shown in Fig. 2 for a fixed $\alpha_{2}=1$. Nonetheless, a larger $\alpha_{1}$ will cause more severe oscillations to the sliding mode as demonstrated in an enlarged plot, because the equivalent gain in the steady state is amplified. Hence, $\alpha_{1}$ is chosen as 1.20 to make a compromise. The common sliding modes in terms of variations of $\alpha_{2}$ are depicted in Fig. 3. Clearly, the sliding mode error is decreased with a larger $\alpha_{2}$, but there are minor variations. In such way, $\alpha_{2}$ is chosen as 0.95 ,

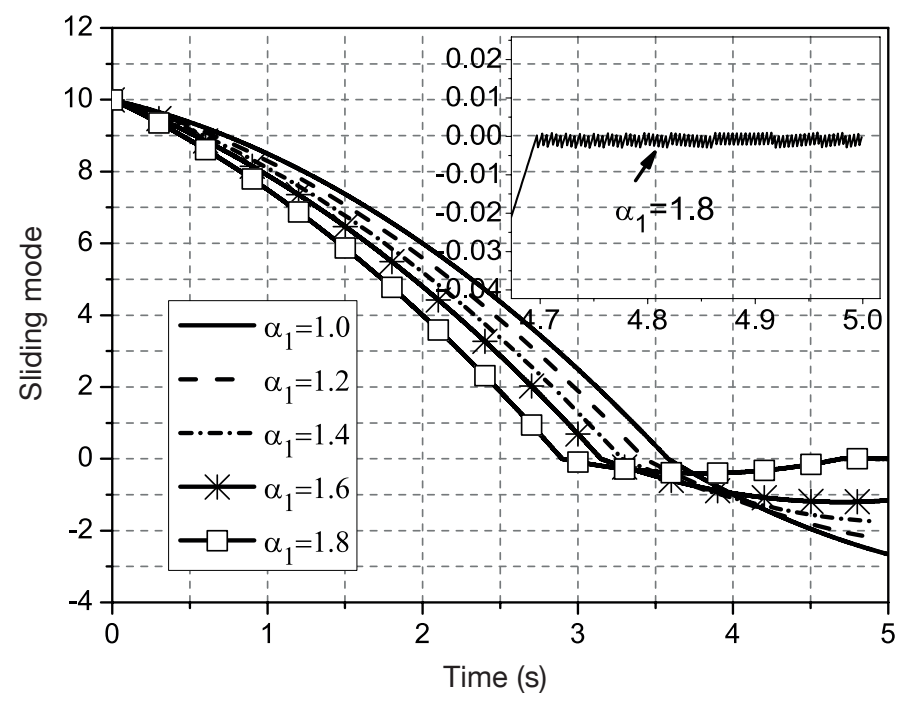

Fig. 2. Common sliding modes with variations of $\alpha_{1}$

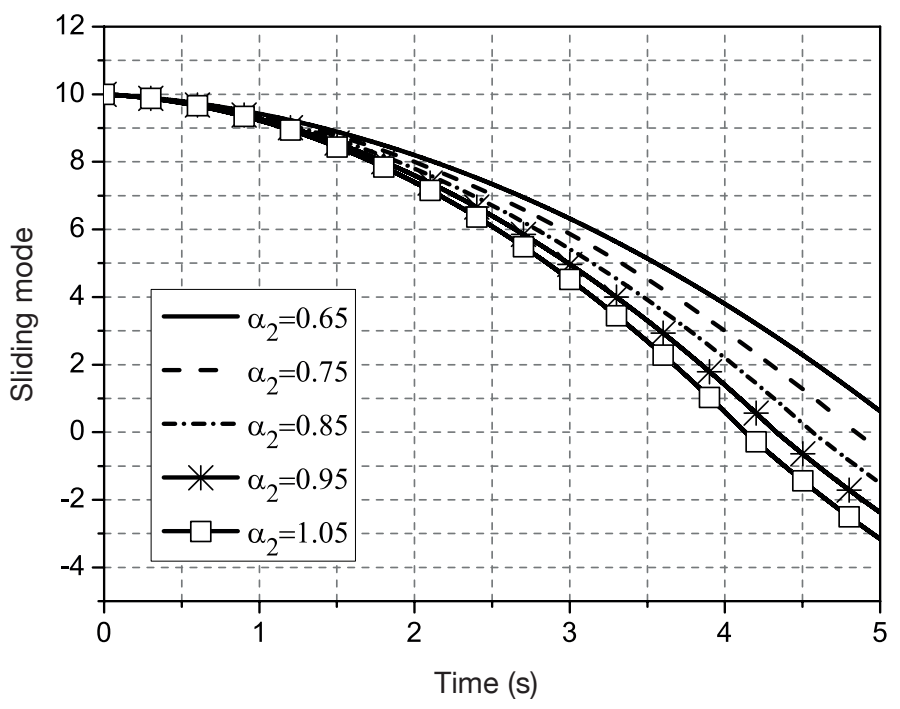

Fig. 3. Common sliding modes with variations of $\alpha_{2}$

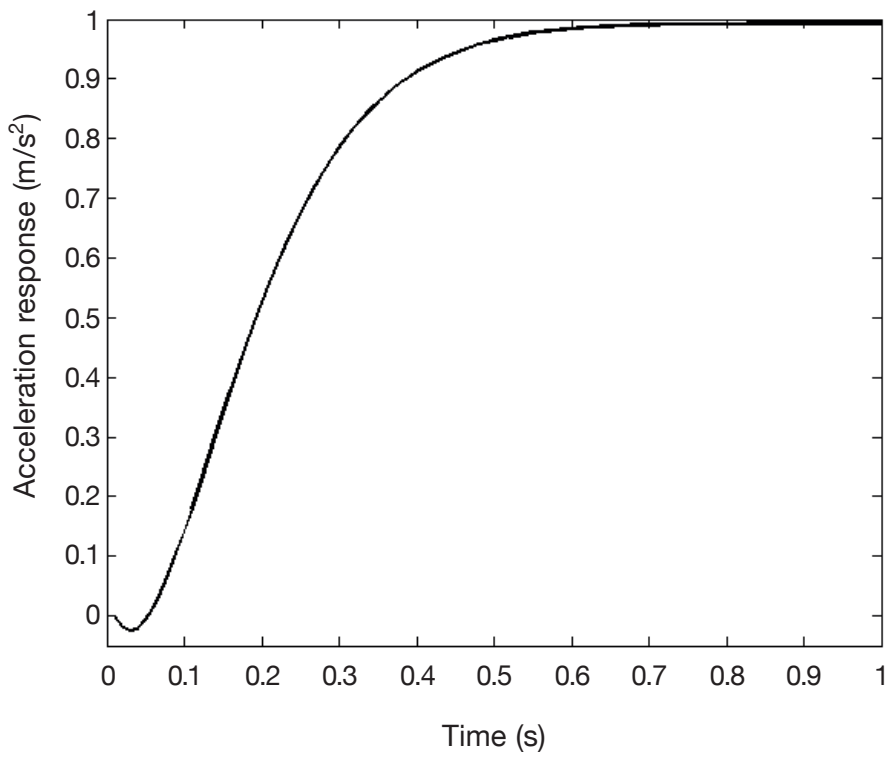

Fig. 4. Step response of the three-loop acceleration control system

approximately based on the parameter selection principle in [11], where $\alpha_{1}=1.5 \sqrt{\varepsilon_{f}}$ and $\alpha_{2}=1.1 \varepsilon_{f}$ with $\varepsilon_{f}=8 \mathrm{~g} / \mathrm{s}$, which assumes that the acceleration of the target can vary $8 \mathrm{~g}$ per second, which is a general specification for the surface-bound missile. Although a high $\omega_{o}$ is helpful for obtaining a small enough error compact set and achieving an accurate estimation, it may cause high-frequency oscillations in practice [19]. Considering both the robustness and the aerodynamic performance requirements, an appropriate trade-off can be achieved by selecting $\omega_{o}=10 \mathrm{rad} / \mathrm{s}$, according to the empirical experience, because the bandwidth of guidance dynamics is much slower than that of aerodynamics in general, and $\omega_{o}=10 \mathrm{rad} / \mathrm{s}$ is sufficient for most flight dynamics [19]. The boundary of $\left|q-\theta_{M}\right|$ in the denominator of (31) is chosen as $\mu=\pi / 2 \times 80 / 90$. To be consistent with [13], $c_{0}$ is chosen as 0.1 . The observer gain $L$ in HOSMO is approximately determined as $L=\varepsilon_{f}$ [13]. Generally, the settling time of the step response in the three-loop acceleration control system needs to be less than one second. Based on this principle, the parameters of the autopilot (49) are tuned as $k_{p}=4.61, k_{i}=2.892, k_{d}=1.34, k_{w}=8.359$ and the control performance can be shown in Fig. 4, which meets the control system specifications.

5.3. Tail chase scenario. The initial positions of the interceptor and the target are set at $(0 \mathrm{~km}, 0 \mathrm{~km})$ and $(3 \mathrm{~km}, 3 \mathrm{~km})$, and the initial flight path angles are $15 \mathrm{deg}$ and $0 \mathrm{deg}$, respectively.

The homing trajectories are shown in Fig. 5. The miss distances are $0.029 \mathrm{~m}$ and $0.055 \mathrm{~m}$ for the FOLO and HOSMO-based methods, respectively. Although the miss distances of these two methods are very similar, the normalised accelerations in the proposed method are smaller and smoother than those of the HOSMO-based method, as depicted in Fig. 6. These commands lead to different elevator deflection needs, as shown in Fig. 7. Here, the limits of the elevator deflection and its rate are given as $25^{\circ}$ and $200^{\circ} / \mathrm{s}$, respectively, a specification com- 


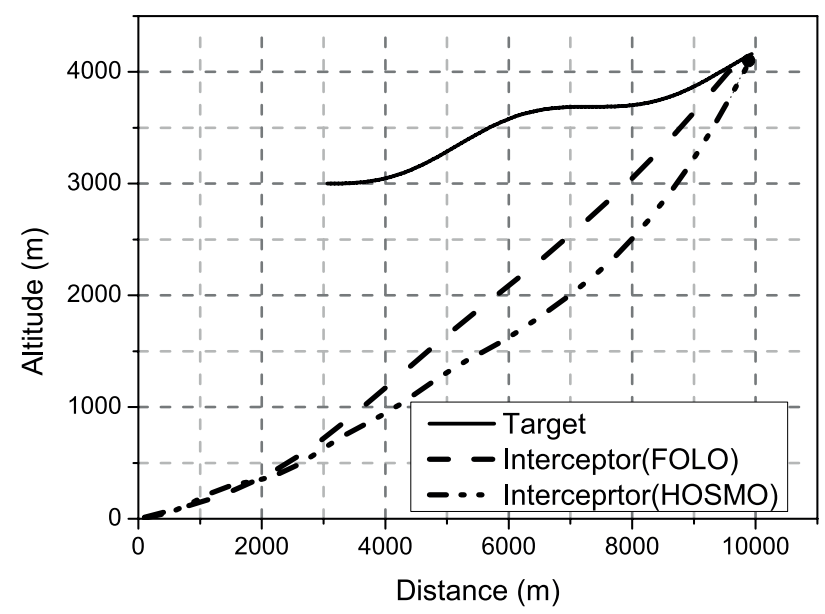

Fig. 5. Homing trajectories in the tail chase scenario

(a) elevator deflection angle

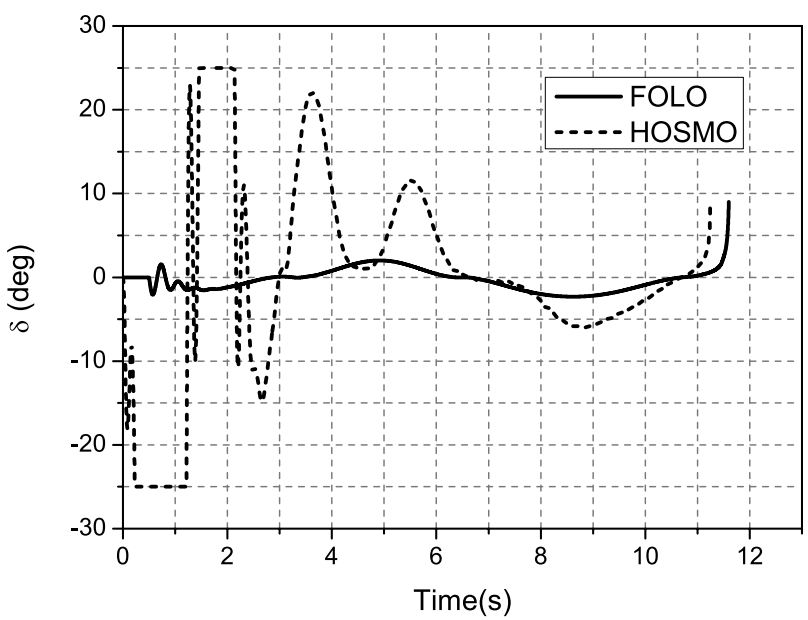

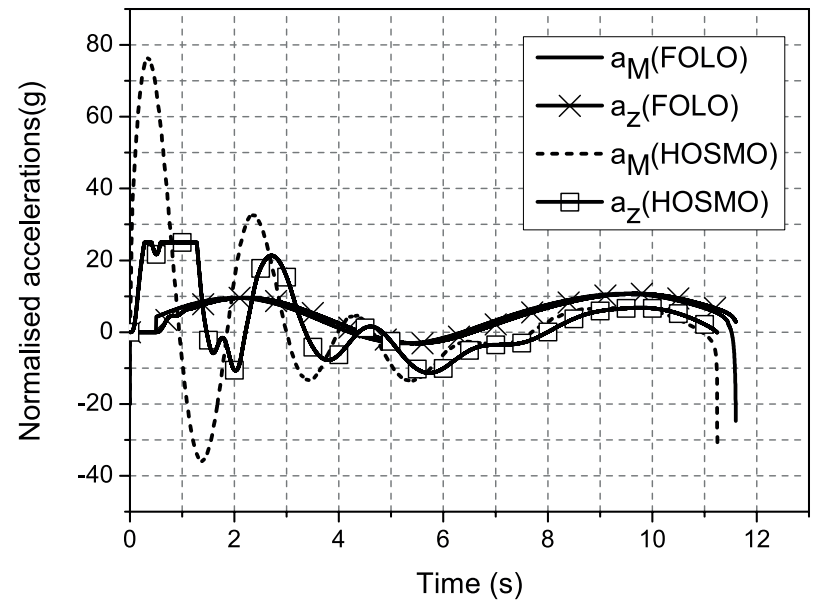

Fig. 6. Normalised missile accelerations in the tail chase scenario

(b) elevator deflection rate

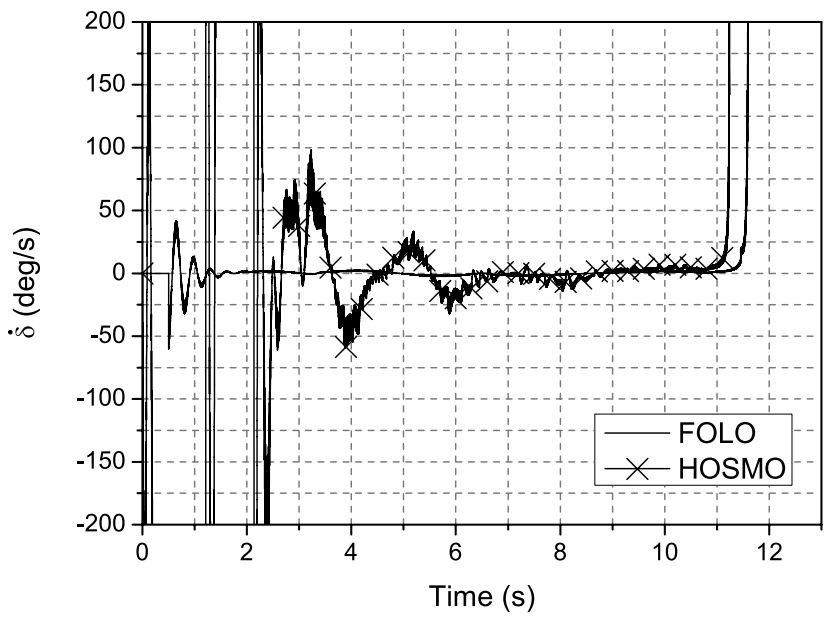

Fig. 7. Elevator responses in the tail chase scenario

bination for the conventional interceptor at present. It is evident that the proposed method has significantly weak requirements for both indices, which are quite favourable for practical implementation because a lower performance actuator is sufficient for the objective such that the research, development, and manufacturing costs can be reduced. Now we attempt to seek the reason behind this key difference. Both methods differ only in the selection of observers. Hence, the sole source of the difference is the observer. To further investigate this, the estimation from the previous HOSMO is illustrated in Fig. 8, where the oscillatory response can be clearly observed during the initial phase, even with the recommended optimal observer gains of [13]. This phenomenon can also be found in [13] with a large amplitude of oscillation. As stated in [31], the homogeneity of the HOSMO, which has been proved in Theorem 1, gives rise to a slow convergent rate of HOSMO when the initial observer errors are large. Although the fractional power in this homogeneous observer is less than 1, which can ensure finite-time convergence, "it makes the convergence time grow unbounded

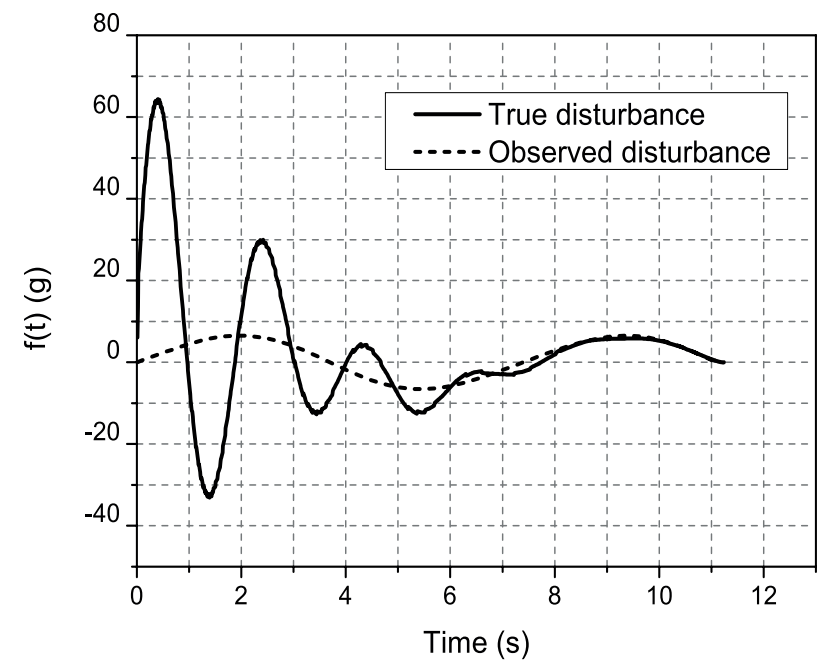

Fig. 8. Disturbance estimation based on HOSMO in the tail chase scenario 


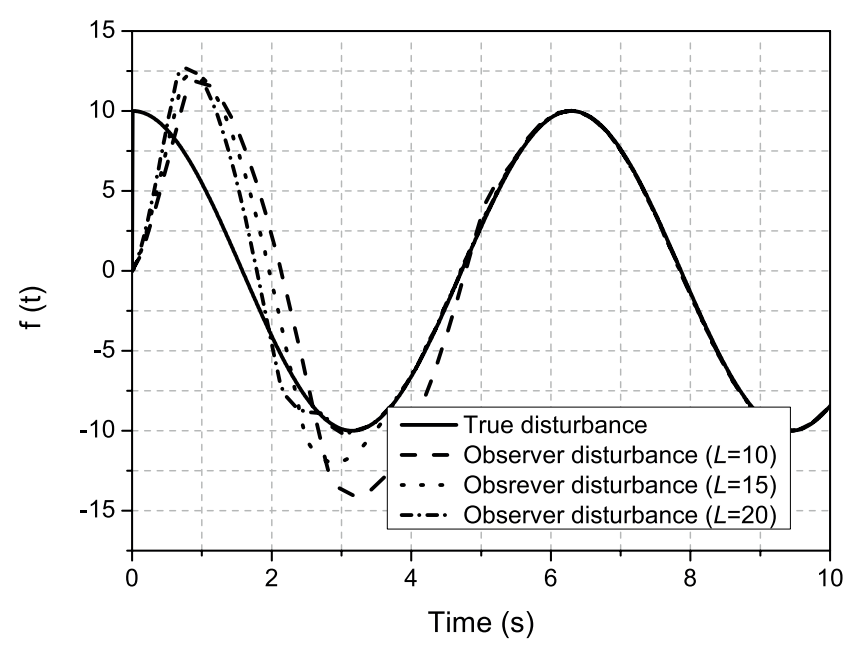

Fig. 9. Simulation results of HOSMO with $f(t)=10 \cos t$

as the initial observer errors grow" [26]. Therefore, a uniform convergence cannot be achieved in HOSMO. Moreover, the simulations for HOSMO estimations, apart from the guidance dynamics, with respect to different observer gains, are shown in Fig. 9. It is evident that the convergent rate cannot be significantly raised, even if the observer gain $L$ is greatly increased, which limits the application of HOSMO.

With regard to the proposed FOLO, the combination of (24) and (44) yields

$$
\dot{e}=\left(-\omega_{o}\right) e+w,
$$

where $e=f-z_{2}$. Hence, one can obtain

$$
e(t)=e^{-\omega_{o} t} e(0)+\int_{0}^{t} e^{-\omega_{o}(t-\tau)} \mathcal{w} d \tau .
$$

It is evident that the convergent rate of FOLO is mainly affected by the observer gain $\omega_{o}$. Meanwhile, since FOLO is a linear observer, it possesses the inherent characteristic of insensitivity

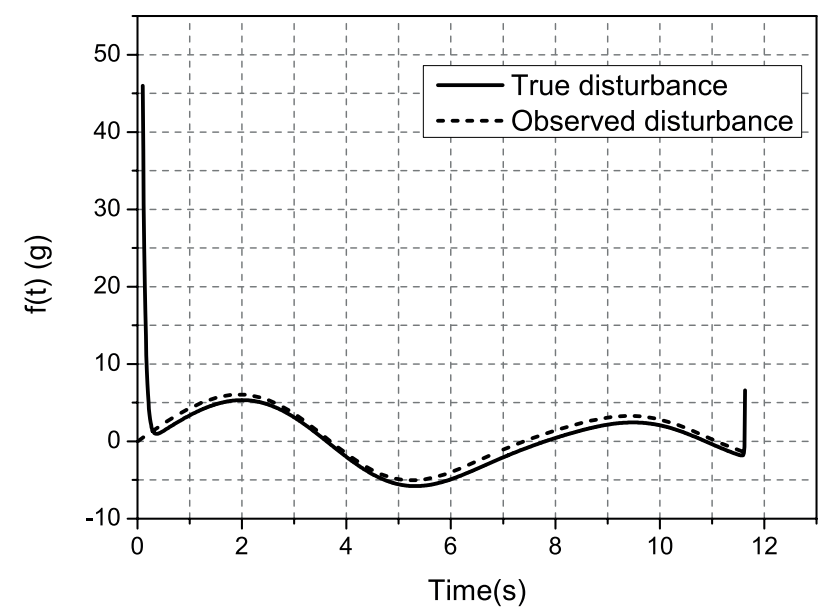

Fig. 10. Disturbance estimation based on FOLO in the tail chase scenario

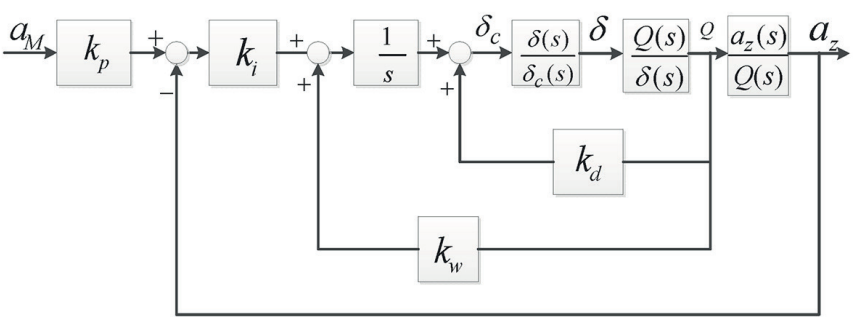

Fig. 11. Block diagram of three-loop acceleration control configuration

to the initial observer errors, which can avoid the oscillation in the initial period, as shown in the utilization of HOSMO. As illustrated in Fig. 10, a uniform estimation of the performance can be generated by using the proposed FOLO.

Thereafter, FOLO can achieve a faster convergent rate and smoother estimation results than HOSMO. Consequently, the smooth and small amplitude guidance command can be generated directly by the FOLO-based method.

Here, we attempt to seek the reason behind the different elevator deflection requirements of these two methods. The block diagram of the three-loop acceleration control configuration is depicted in Fig. 11. The small perturbation theory is used to linearize the longitudinal dynamics (47), and one can obtain the following two transfer functions [19]:

$$
\frac{Q(s)}{\delta(s)}=\frac{a_{1} s+a_{0}}{s^{2}+b_{1} s+b_{0}}
$$

and

$$
\frac{a_{z}(s)}{Q(s)}=\frac{c_{1}}{a_{1} s+a_{0}}
$$

where $a_{0}, a_{1}, b_{0}, b_{1}$ and $c_{1}$ are the characteristic parameters. According to Fig. 11, we can obtain the transfer function from the guidance command $a_{M}$ to the elevator deflection $\delta$ as

$$
\frac{\delta(s)}{a_{M}(s)}=\frac{n_{2} s^{2}+n_{1} s+n_{0}}{d_{4} s^{4}+d_{3} s^{3}+d_{2} s^{2}+d_{1} s+d_{0}},
$$

and its coefficients are

$$
\left\{\begin{array}{l}
n_{2}=k_{p} k_{i} \omega_{a} \\
n_{1}=k_{p} k_{i} \omega_{a} b_{1} \\
n_{0}=k_{p} k_{i} \omega_{a} b_{0} \\
d_{4}=1 \\
d_{3}=b_{1}+\omega_{a} \\
d_{2}=b_{0}+\omega_{a}\left(b_{1}-k_{d} a_{1}\right) \\
d_{1}=\omega_{a}\left(b_{0}-k_{d} a_{0}-k_{w} a_{1}\right) \\
d_{0}=\omega_{a}\left(k_{i} c_{1}-k_{w} a_{0}\right)
\end{array} .\right.
$$

According to (54), the elevator deflection requirement is approximately proportional to the amplitude of $a_{M}$. Since the guid- 
(a) case 1

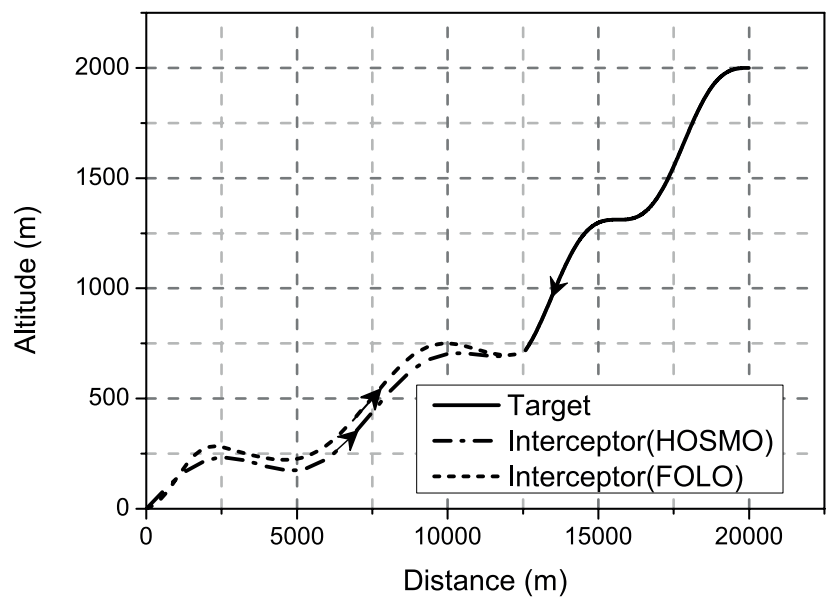

(b) case 2

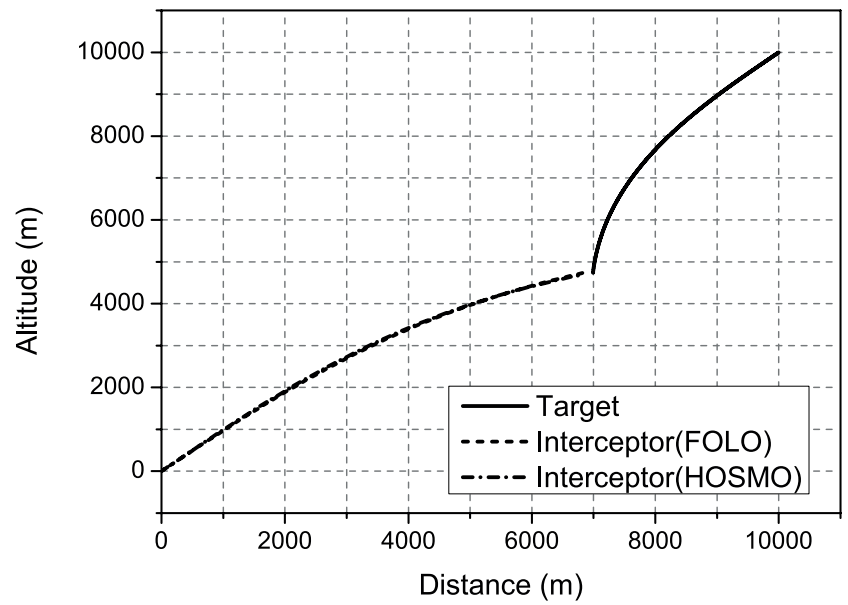

Fig. 12. Homing trajectories in the head-on interception scenario

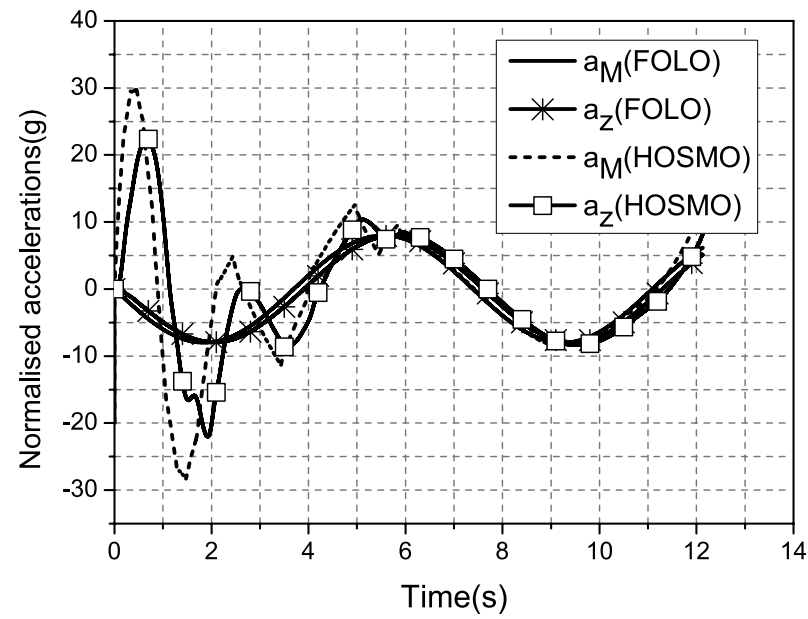

Fig. 13. Normalised missile accelerations in the head-on interception scenario

ance command from the FOLO method is much smaller at the initial period than that of the HOSMO method, the proposed method has a weaker elevator deflection requirement in contrast with the HOSMO-based method.

5.4. Head-on interception scenario. In this sub-section, two head-on interception scenarios are considered. The initial positions and flight path angles of the interceptor and target are listed in Table 2. Like the results shown in Fig. 12, the two guidance laws also obtain similar trajectories in these two cases, respectively. The miss distances are $0.101 \mathrm{~m}$ and $0.085 \mathrm{~m}$ for case 1 , and $0.092 \mathrm{~m}$ and $0.134 \mathrm{~m}$ for case 2, when the FOLO method and the HOSMO-based method are used, respectively. For simplicity, only the results of case 1 are investigated in detail. The normalised missile accelerations are illustrated in Fig. 13 for these two approaches. A weaker requirement of the elevator deflection rate using the proposed method is still maintained, as illustrated in Fig. 14, comparing with the HOSMO-based method. The disturbance estimations using these two observers are shown in Figs. 15 and 16, re-

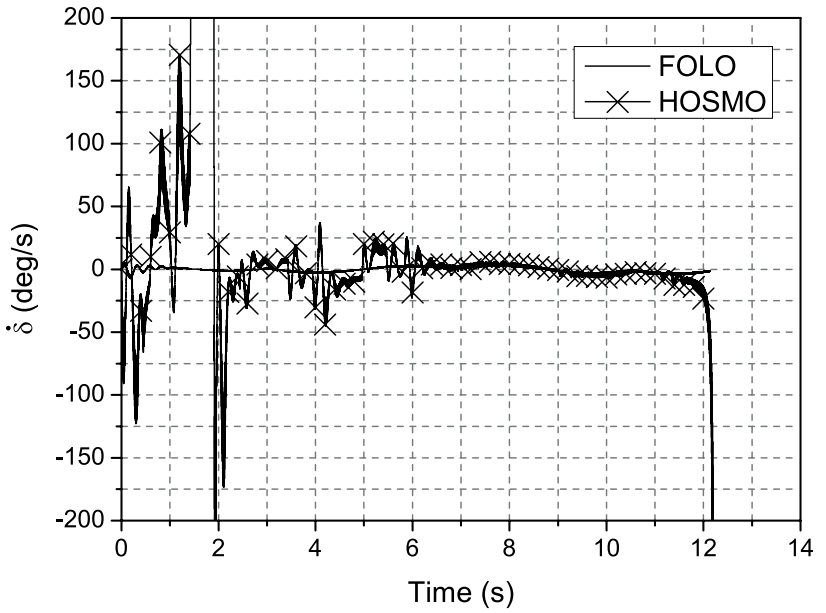

Fig. 14. Elevator deflection rate responses in the head-on interception scenario

spectively, which are consistent with the analysis results of section 5.3.

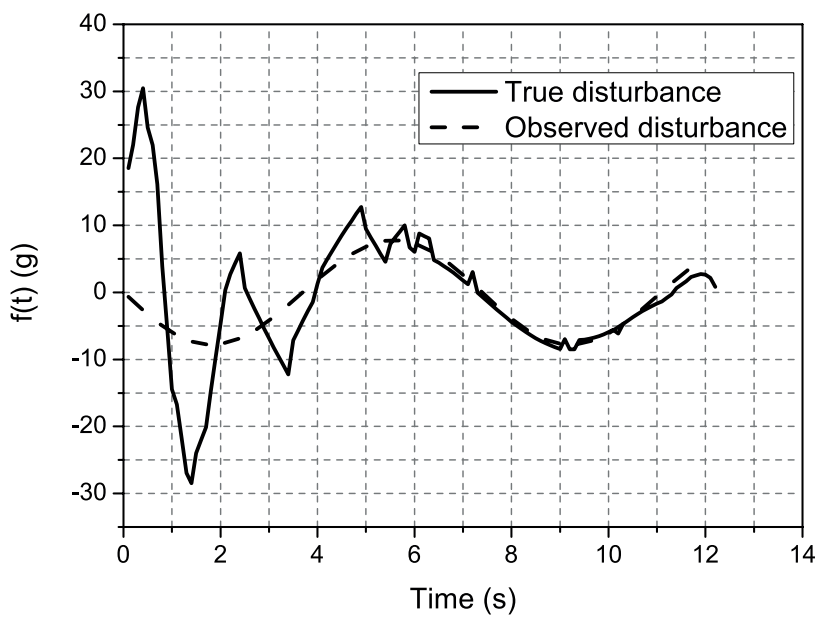

Fig. 15. Disturbance estimation based on HOSMO in the head-on interception scenario 
Table 2

Initial information

\begin{tabular}{|c|c|c|c|}
\hline \multicolumn{2}{|c|}{ Case 1 } & \multicolumn{2}{c|}{ Case 2 } \\
\hline Interceptor & Target & Interceptor & Target \\
\hline $\begin{array}{c}(0 \mathrm{~km}, 0 \mathrm{~km}) \\
8 \mathrm{deg}\end{array}$ & $\begin{array}{c}(20 \mathrm{~km}, 2 \mathrm{~km}) \\
180 \mathrm{deg}\end{array}$ & $\begin{array}{c}(0 \mathrm{~km}, 0 \mathrm{~km}) \\
45 \mathrm{deg}\end{array}$ & $\begin{array}{c}(10 \mathrm{~km}, 10 \mathrm{~km}) \\
-135 \mathrm{deg}\end{array}$ \\
\hline
\end{tabular}

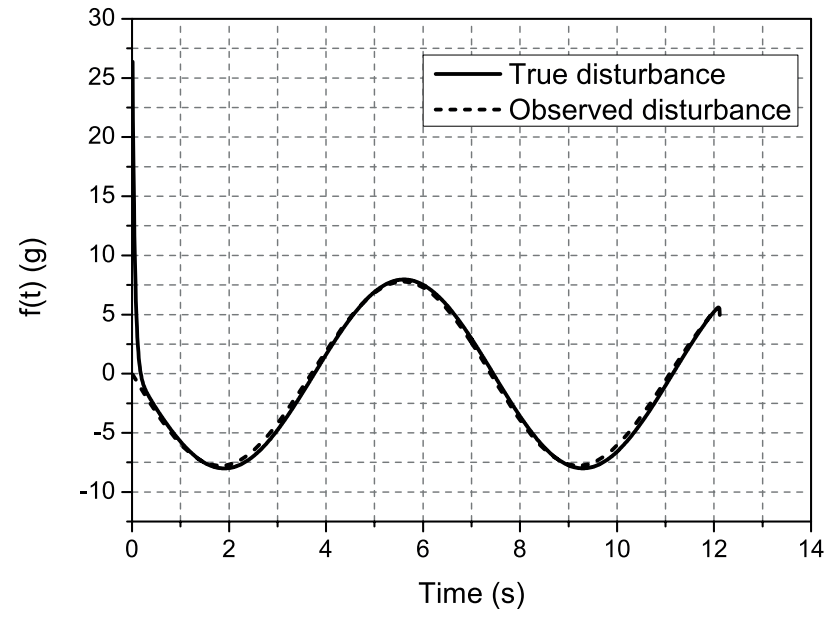

Fig. 16. Disturbance estimation based on FOLO in the head-on interception scenario

5.5. Target manoeuvre with bang-bang acceleration. Assume $a_{T}=8 \operatorname{sign}(\sin (0.85 t)) \mathrm{g}$. The initial positions of the interceptor and the target are set at $(0 \mathrm{~km}, 0 \mathrm{~km})$ and $(4.5 \mathrm{~km}, 4.5 \mathrm{~km})$, and the initial flight path angles are $10 \mathrm{deg}$ and $25 \mathrm{deg}$, respectively. The homing trajectories are depicted in Fig. 17 for the FOLO method and the HOSMO-based method with the miss distances of $0.132 \mathrm{~m}$ and $0.205 \mathrm{~m}$, respectively. As shown in Fig. 18, the oscillatory response in the estimation results can still be found during the initial phase of the HOSMO-based method, leading to large amplitudes of normalised missile acceleration and fast elevator deflection as depicted in Figs. 19 and 20 respectively.

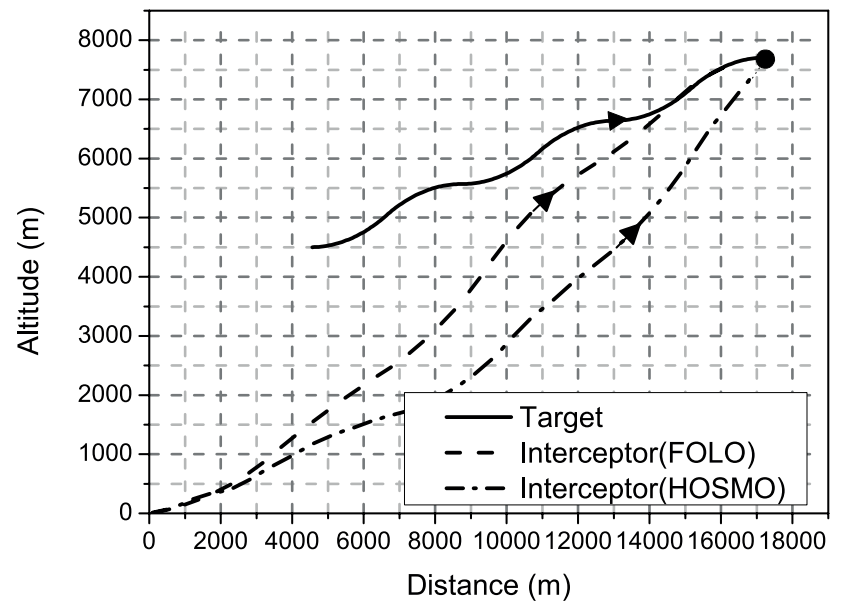

Fig. 17. Homing trajectories with a bang-bang manoeuvring target

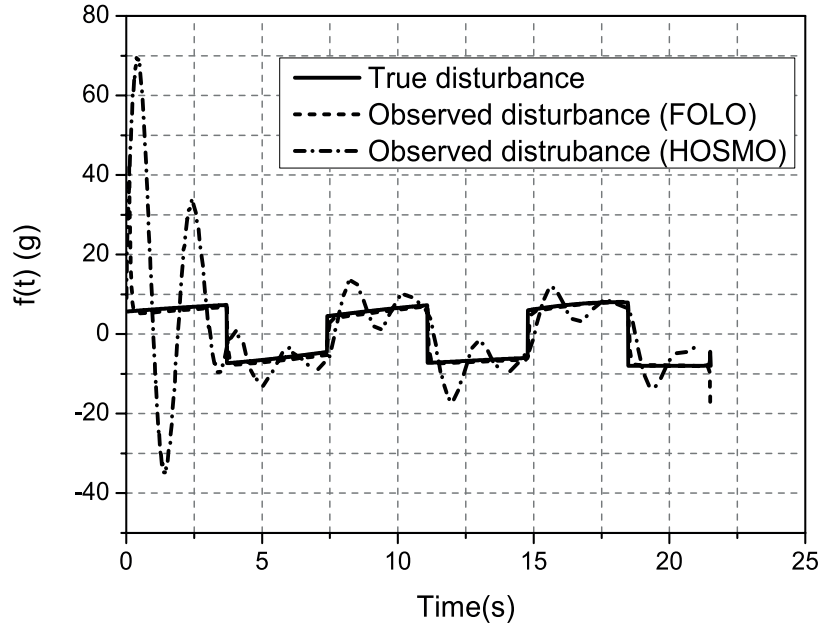

Fig. 18. Disturbance estimations with a bang-bang manoeuvring target

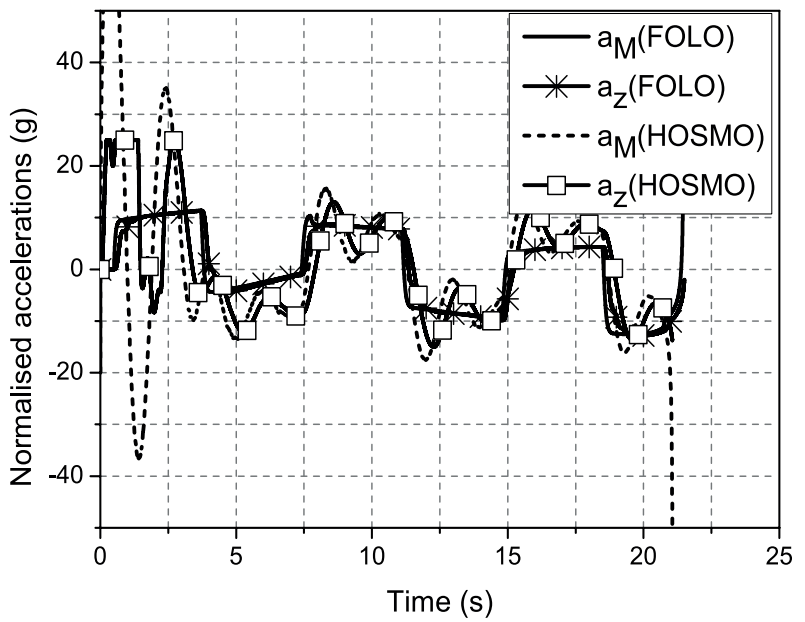

Fig. 19. Normalised missile accelerations with a bang-bang manoeuvring target

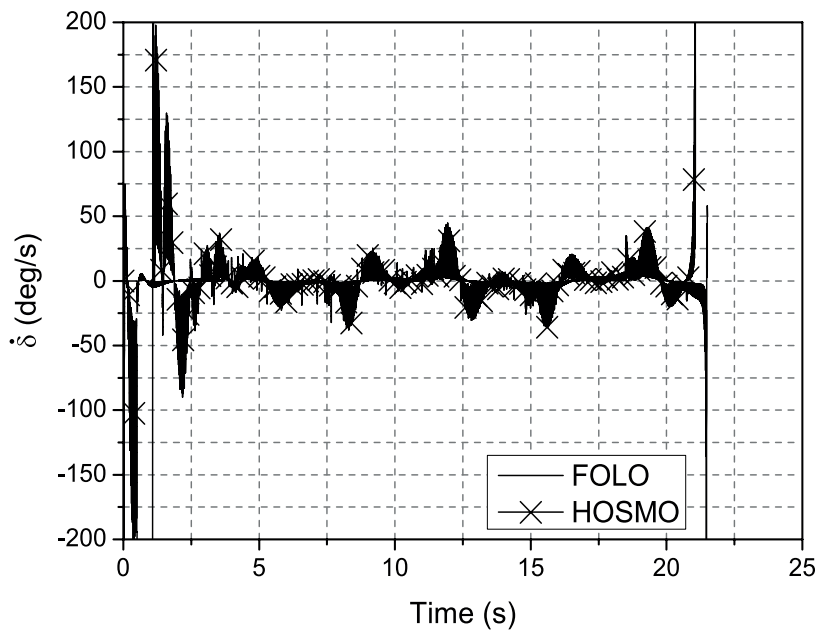

Fig. 20. Elevator deflection rate responses with a bang-bang manoeuvring target 
Generally, the guidance system is unacceptable if it is fairly sensitive to the measurement noises on LOS rate. To test whether the systems are sensitive to the noise, we assume that the LOS rate has additional white noises as its measurement noises with an intensity of $2 \% \mathrm{~s}$. The responses of the elevator deflection angle are shown in Fig. 21 for these two methods. It is evident that both methods have the identical level of noise sensitivities. This is because $\dot{q}$ is not entirely contained in the disturbance $f(t)$, and the observer has no effect on the noise filtering for $\dot{q}$. As depicted in (14), $\dot{q}$ explicitly exists in the guidance command in a straightforward manner no matter what observers are employed. Therefore, the FOLO-based and HOSMO-based methods have comparable sensitivities to the measurement noises on $\dot{q}$. As a result, the applicability of the FOLO-based guidance law is not weaker than the original nonlinear observer based one.

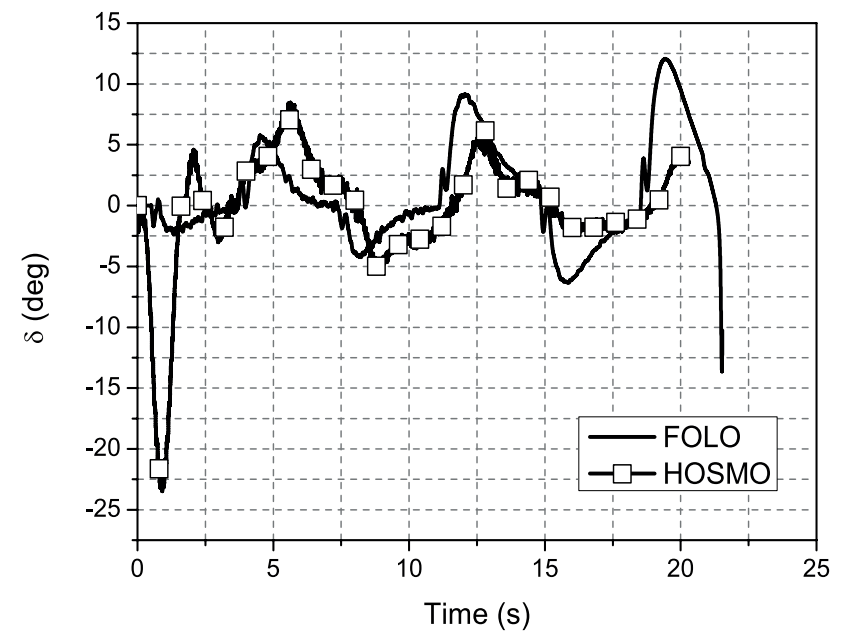

Fig. 21. Elevator deflection responses with measurement noises on the LOS rates

\section{Conclusion}

In this paper, a FOLO-aided guidance law, which can be used to intercept highly manoeuvrable targets, was presented. The second-order sliding mode methodology based on a super-twisting algorithm was utilised to design the guidance law. Meanwhile, one FOLO was designed to estimate the target manoeuvre, which can achieve a uniform estimation performance compared with the original nonlinear observer. The entire linear observer-based design has weaker requirements of the elevator deflection than the previous nonlinear observer-based method. The reason for the differences between these two observer-based methods was thoroughly investigated. Moreover, the closed-loop system is uniformly ultimately bounded (UUB) when the FOLO is used. The comparative numerical results show the effectiveness of the guidance law with the linear observer. This design may provide a practical solution for practitioners.
Acknowledgements. This work was supported in part by the Natural Science Foundation of China under grants of 61573197, 61603051, 61603056, and 61573199, the Tianjin Natural Science Foundation (Grant Nos. 14JCYBJC18700 and 13JCYBJC17400), the Research Fund of Tianjin Key Laboratory of Process Measurement and Control under Grant No. TKLPMC-201613, and NRF Grant Competitive Programme for Rated Researchers (CPRR) (Grant No. 93539).

\section{REFERENCES}

[1] P. Zarchan, Tactical and Strategic Missile Guidance, American Institute of Aeronautics and Astronautics Inc, Virginia, 1997.

[2] F. Imado and S. Miwa, "Missile guidance algorithm against high-g barrel roll maneuvers", J. of Guidance, Control, and Dynamics 17 (1), 123-128 (1994).

[3] N. Cho and Y. Kim, "Optimality of augmented ideal proportional navigation for maneuvering target interception", IEEE Trans. on Aerospace Electronic Systems 52 (2), 948-954 (2016).

[4] H. Cho, C.K. Ryoo, and M.J. Tahk, "Closed-form optimal guidance law for missiles of time-varying velocity", J. of Guidance, Control, and Dynamics 19 (5), 1017-1022 (1996).

[5] R. Gitizadeh, I. Yaesh, and J.Z. Ben-Asher, "Discrete-time optimal guidance", J. of Guidance, Control, and Dynamics 22 (1), 171-174 (1999).

[6] C.D. Yang and H.Y. Chen, "Three-dimensional nonlinear $\mathrm{H} \infty$ guidance law", International J. of Robust and Nonlinear Control 11 (2), 109-129 (2001).

[7] S. Kamal, J.A. Moreno, A. Chalanga, B. Bandyopadhyay, and L.M. Fridman, "Continuous terminal sliding-mode controller", Automatica 69, 308-314 (2016).

[8] W.H. Wang, S.F. Xiong, X.D. Liu, S. Wang, and L.J. Ma, “Adaptive nonsingular terminal sliding mode guidance law against maneuvering targets with impact angle constraint", Proc.of IMechE, Part G: J Aerospace Engineering 229 (5), 867-890 (2015).

[9] A. Levant, "Sliding order and sliding accuracy in sliding mode control", International J. of Control 58 (6), 1247-1263 (1993).

[10] G. Bartolini, A. Ferrara, and E. Usani, "Chattering avoidance by second-order sliding mode control", IEEE Trans. on Automatic Control 43 (2), 241-246 (1998).

[11] A. Levant, "Higher-order sliding modes, differentiation and output-feedback control", International J. of Control 76 (9-10), 924-941 (2003).

[12] A. Levant, "Robust exact differentiation via sliding mode technique", Automatica 34 (3), 379-384 (1998).

[13] Y.B. Shtessel, I.A. Shkolnikov, and A. Levant, "Smooth second-order sliding modes: Missile guidance application", Automatica 43 (8), 1470-1476 (2007).

[14] Y.B. Shtessel, I.A. Shkolnikov, and A. Levant, "Guidance and control of missile interceptor using second-order sliding modes", IEEE Trans. on Aerospace Electronic Systems 45 (1), 110-124 (2009).

[15] J.Q. Han, "From PID to active disturbance rejection control", IEEE Trans. on Industrial Electronics 56 (3), 900-906 (2009).

[16] Z.Q. Gao, "Scaling and bandwidth-parameterization based controller tuning", American Control Conf., 4989-4996, (2003).

[17] Q.X. Wu, M.W. Sun, Z.H. Wang, and Z.Q. Chen, "Practical solution to efficient flight path control for hypersonic vehicle", Trans. of the Japan Society for Aeronautical and Space Sciences 59 (4), 195-204 (2016). 
[18] M. Przybyla, M. Kordasz, R. Madonski, P. Herman, and P. Sauer,. "Active disturbance rejection control of a 2DOF manipulator with significant modeling uncertainty", Bull. Pol. Ac:. Tech. 60 (3), 509-520 (2012).

[19] M.W. Sun, Z.H. Wang, and Z.Q. Chen, "Practical solution to attitude control within wide envelope", Aircraft Engineering Aerospace Technology 86 (2), 117-128 (2014).

[20] S.E. Talole, A.A. Godbole, and J.P. Kolhe, "Robust roll autopilot design for tactical missiles", J. of Guidance, Control, and Dynamics 34 (1), 107-117 (2011).

[21] D.M. Qiu, M.W. Sun, Z.H. Wang, Y.K. Wang, and Z.Q. Cheng, "Practical wind disturbance rejection for large deep space observatory antenna", IEEE Trans. on Control System Technology 22 (5), 1983-1990 (2014).

[22] A. Aguilera-González, C.M. Astorga-Zaragoza, M. Adam-Medina, D. Theilliol, J. Reyes-Reyes, C.-D. Garcia-Beltran, "Singular linear parameter-varying observer for composition estimation in a binary distillation column", IET Control Theory and Applications 7 (3), 411-422 (2013).

[23] A. Mokhtari, N.K. M'Sirdi, K. Meghriche, and A. Belaidi, "Feedback linearization and linear observer for a quadrotor unmanned aerial vehicle", Advanced Robotics 20 (1), 71-79 (2006).
[24] A. Bacciotti and L. Roiser, Lyapunov Functions and Stability in Control Theory, Springer-Verlag, New York, 2011.

[25] I.A. Shkolnikov, Y.B. Shtessel, and D. Lianos, "Integrated guidance-control system of a homing interceptor: sliding mode approach", AIAA Guidance, Navigation, and Control conf. and Exhibit, 1-11 (2001).

[26] M.T. Angulo, J.A. Moreno, and L. Fridman, "Robust exact uniformly convergent arbitrary order differentiator", Automatica, 49 (8), 2489-2495 (2013).

[27] J.I. Lee, I.S. Jeon, and M.J. Tahk, "Guidance law to control impact time and angle", IEEE Trans. on Aerospace Electronic Systems 43 (1), 301-310 (2007).

[28] M.W. Sun, Q. Xu, S.Z. Du, Z.Q. Chen, and D.X. Zhang, "Practical solution to impact angle control in vertical plane", $J$. of Guidance, Control, and Dynamics 37 (3), 1022-1027 (2014).

[29] H.K. Khalil, Nonlinear Systems, Prentice Hall, New Jersey, 1996.

[30] C.P. Mracek and D.B. Ridgely, "Missile longitudinal autopilots: comparison of multiple three loop topologies", AIAA Guidance, Navigation, and Control conf. and Exhibit, 917-928 (2005).

[31] A. Levant, "Non-homogeneous finite-time-convergent differentiator", IEEE Conf. on Decision and Control and Chinese Control Conf., 8399-8404 (2009). 